\title{
Demonstration of a widely-tunable and fully-coherent high-gain harmonic-generation free-electron laser
}

\author{
B. Liu, ${ }^{1}$ W. B. Li, ${ }^{2}$ J. H. Chen, ${ }^{1}$ Z. H. Chen, ${ }^{1}$ H.X. Deng, ${ }^{1}$ J. G. Ding, ${ }^{1}$ Y. Fan, ${ }^{1}$ G. P. Fang, ${ }^{1}$ C. Feng, ${ }^{1}$ L. Feng, ${ }^{1}$ Q. Gu, ${ }^{1}$ \\ M. Gu, ${ }_{1}^{1}$ C. Guo, ${ }^{1}$ D. Z. Huang, ${ }^{1}$ M. M. Huang, ${ }^{1}$ W. H. Huang, ${ }^{3}$ Q. K. Jia, ${ }^{4}$ T. H. Lan, ${ }^{1}$ Y. B. Leng, ${ }^{1}$ D. G. Li, ${ }^{1}$ W. M. Li, ${ }^{4}$ \\ X. Li, ${ }_{1}^{1}$ G. Q. Lin, ${ }^{1}$ L. Shang, ${ }^{4}$ L. Shen, ${ }^{1}$ C.X. Tang, ${ }^{3}$ G. L. Wang, ${ }^{1}$ L. Wang, ${ }^{1}$ R. Wang, ${ }^{1}$ X. T. Wang, ${ }^{1}$ Zhan S. Wang, ${ }^{2}$ \\ Zhi S. Wang, ${ }^{1}$ H. F. Yao, ${ }^{1}$ K. R. Ye,${ }^{1}$ L.X. Yin, ${ }^{1}$ L. Y. Yu, ${ }^{1}$ J. Q. Zhang, ${ }^{1}$ Meng Zhang, ${ }^{1}$ Miao Zhang, ${ }^{1}$ T. Zhang, ${ }^{1}$ \\ W. Y. Zhang, ${ }^{1}$ S. P. Zhong, ${ }^{1}$ Q. G. Zhou, ${ }^{1}$ D. Wang, ${ }^{1}$ and Z. T. Zhao ${ }^{1, *}$ \\ ${ }^{1}$ Shanghai Institute of Applied Physics, Chinese Academy of Sciences, Shanghai 201800, China \\ ${ }^{2}$ Key Laboratory of Advanced Micro-Structured Materials, MOE, Institute of Precision Optical Engineering, \\ Department of Physics, Tongji University, Shanghai 200092, China \\ ${ }^{3}$ Department of Engineering Physics, Tsinghua University, Beijing 100084, China \\ ${ }^{4}$ National Synchrotron Radiation Laboratory, Hefei, Anhui 230029, China
}

(Received 13 August 2012; published 19 February 2013)

\begin{abstract}
Free-electron lasers (FELs) are fourth-generation light sources that deliver extremely high intensity, ultrashort light pulses over a broad wavelength range from far-infrared to hard $\mathrm{x}$ ray. FELs based on the self-amplified spontaneous emission principle have been successfully operated with ultrahigh brightness and a broad wavelengths tuning range with good transverse coherence but poor temporal coherence. In contrast, the laser-seeded FELs have provided full coherence but at selected central wavelengths, usually the harmonics of the laser seeds, with relatively narrower tuning range. We report the experimental demonstration of a high-gain harmonic-generation (HGHG) FEL that is continuously tunable over a wide range using the combination of optical parametrical amplification, variable-gap undulator, and harmonic selection, where the temporal coherence is preserved as confirmed with the Michelson interferometry. In order to achieve higher photon energies, the first try of cascaded HGHG with a fresh-bunch technique is also made at the Shanghai Deep Ultraviolet Free-electron Laser test facility.
\end{abstract}

DOI: 10.1103/PhysRevSTAB.16.020704

PACS numbers: $41.60 . \mathrm{Cr}$

\section{INTRODUCTION}

High-gain free-electron lasers (FELs), serving as high-intensity coherent light sources, are being actively developed around the world. Most of the FELs in the x-ray wavelengths [1-4] are based on the self-amplified spontaneous emission (SASE) [5,6] principle. SASE FEL can provide extremely high-intensity, ultrashort light pulses with good spatial coherence [7-11] but poor temporal coherence [9-13], since it starts up from shot noise of the electron beam [14].

Coherence is one of the most distinguishing features of a laser source. It has very important applications in many fundamental research fields, especially coherent diffraction imaging and holography. To improve the temporal coherence of SASE-FEL radiation, various seeded FEL schemes have been proposed and demonstrated, including external seeding [15-26] or self-seeding [27-29].

Another significant feature of an FEL, especially important for serving different users or element-specific

\footnotetext{
*zhaozt@sinap.ac.cn
}

Published by the American Physical Society under the terms of the Creative Commons Attribution 3.0 License. Further distribution of this work must maintain attribution to the author(s) and the published article's title, journal citation, and DOI. spectroscopy experiments, lies in its flexible wavelength tunability, which has been realized on SASE based FEL facilities. The resonance condition for a free-electron laser is as follows:

$$
\lambda=\frac{\lambda_{u}}{2 \gamma^{2}}\left(1+\frac{K^{2}}{2}\right)
$$

where $\lambda$ is the FEL radiation wavelength, $\lambda_{u}$ is the period length of undulator, $\gamma$ is the relativistic factor of electron beam, and $K$ is the dimensionless undulator parameter. One can vary the wavelength of the FEL output by changing the electron beam energy or undulator gap or both. However, changing beam energy usually involves variation of most parameters, relative strengths of the magnets, hence the beam optics, and the FEL amplification process.

For seeding schemes, this is even more complicated. In the typical high-gain harmonic-generation (HGHG) [15] configuration, the output wavelength of the FEL is determined by the wavelength of the laser seed and the resonant wavelength of the radiator. Therefore, to alter the output wavelength of an HGHG FEL, the seed laser should be tunable and the related electron beam parameters must be changed accordingly. Over the past decade, it was believed that it would be very hard to tune the wavelength of seeded FELs because tuning wavelength of the seed was a timeand effort-consuming procedure. 
An alternative tuning method for seeded FELs has been demonstrated at the DUV-FEL@NSLS utilizing chirped electron beam [30]. One weakness of this method is that dealing with energy chirp of the electron beam will change beam-related optics and many other parameters. Another method by utilizing very wide-bandwidth laser seed is also proposed and demonstrated [31-35]. However, the tuning ranges of both methods are very limited, only a few percent of the central wavelength.

In the recent years, there are many rapid progresses of laser technologies, especially the optical parametric amplification (OPA) $[36,37]$ technology that makes tuning of the laser wavelength fairly easy and quick. Thus, combining advanced OPA technology with variable-gap undulators and HGHG technique, a fully-tunable seeded FEL becomes possible and has been recently demonstrated at the Shanghai Deep Ultraviolet Free-electron Laser test facility (SDUV-FEL) [38].

\section{HGHG TUNING EXPERIMENTS}

The SDUV-FEL is a multipurpose test facility for FEL principle studies, capable of testing SASE, HGHG, cascaded HGHG, echo-enabled harmonic generation (EEHG) [19], etc. The schematic layout of SDUV-FEL for HGHG experiments is shown in Fig. 1. The main parameters used during the tuning experiments can be found in Table I.

The laser seed comes from the commercial coherent Ti: Sa system followed by a traveling wave optical parametric amplifying system, which can provide up to a few microJoules energy with about 100 fs pulse duration and wavelengths continuously tunable from 1160 to $1580 \mathrm{~nm}$.

The modulator, a ten-period variable-gap permanent magnet undulator (PMU) with the period length of $50 \mathrm{~mm}$, is set to fulfill the resonant condition with electron beam energy and laser seed. The radiator section, consisting of two variable-gap PMUs with 40 periods of $40 \mathrm{~mm}$ period length, is set in such a way that the FEL wavelength is the harmonic of laser seed while fulfilling the resonant condition. During the experiments, the beam energy at the exit of the electron linac is set to $148.0 \mathrm{MeV}$. With a harmonic up-conversion number from 2 to 4, the FEL radiation can cover the wavelength region between 290 and $790 \mathrm{~nm}$.

The output FEL signal is maximized by optimizing the resonance conditions of the undulators, dispersive

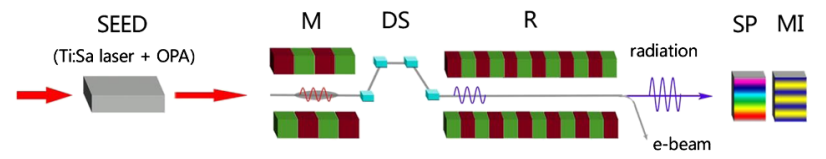

FIG. 1. Schematic of the SDUV-FEL (SEED is the external laser seed, which consists of a Ti:Sa laser system followed by an OPA, $M$ is the variable-gap modulator section, DS is the dispersive section, $\mathrm{R}$ is the variable-gap radiator section, SP is the spectrograph, and MI is the Michelson interferometer).
TABLE I. Main parameters of the SDUV-FEL.

\begin{tabular}{lcc}
\hline \hline Parameter & Value & Unit \\
\hline Electron beam & & \\
Beam energy & 148.0 & $\mathrm{MeV}$ \\
Projected energy spread & $0.2 \%$ & \\
Charge & $100-300$ & $\mathrm{pC}$ \\
Normalized emittance & $4-6$ & $\mathrm{~mm} \mathrm{mrad}$ \\
Pulse length (FWHM) & $2-8$ & $\mathrm{ps}$ \\
\hline Seed laser & & $\mathrm{nm}$ \\
Wavelength & $1160-1580$ & $\mathrm{fs}$ \\
Pulse length (FWHM) & $\sim 100$ & $\mu \mathrm{J}$ \\
Pulse energy & $0-10$ & $\mathrm{~mm}$ \\
\hline Modulator & & \\
Period length & 50 & $\mathrm{~mm}$ \\
Period number & 10 & $\mathrm{~mm}$ \\
Undulator parameter $K$ & $2.0-3.0$ & \\
\hline Dispersive section & & \\
Dispersive strength $\left(\mathrm{R}_{56}\right)$ & $0-3.0$ & \\
\hline Radiator & & \\
Period length & 40 & \\
Period number & $0.9-2.5$ & \\
Undulator parameter $K$ & & \\
\hline \hline
\end{tabular}

strength, and the beam orbits in the FEL amplifier system. The spectrum is measured by an Ocean QE65000 spectrometer which is capable of detecting the signal from 350 to $1100 \mathrm{~nm}$. By changing the wavelength of the OPA and undulator gaps gradually while keeping the FEL resonance conditions, the wavelength of the FEL signal can be changed continuously and smoothly, as shown in Fig. 2(a) (in this case radiating at the second harmonic, and only few typical cases drawn). The gaps of the radiator magnets are then set to produce the 3rd harmonic of the seed. The FEL wavelengths of 387-527 nm are obtained by scanning the OPA wavelength, shown in Fig. 2(b). The fourth harmonic is also measured and gives $350-395 \mathrm{~nm}$ output (here the lower limit is restricted by the spectrometer), as in Fig. 2(c).

The electron beam from the linac accelerator is kept unchanged during the FEL experiments. Therefore, many potential troublesome processes such as phase shifting, high voltage tuning, orbit correction, etc. are avoided. Tunability is obtained by varying the OPA output wavelength, changing undulator gaps, and adjusting only a few correction elements in the radiation section. These parameters are optimized at many wavelengths, and then all the corresponding values are stored into a preset table. Once this is done, the tuning process for each wavelength takes place with only one knob and within several seconds. Setting the OPA wavelength and changing magnets strength are very fast, and the tuning speed is mainly limited by the undulator gap motion. Certainly even more 

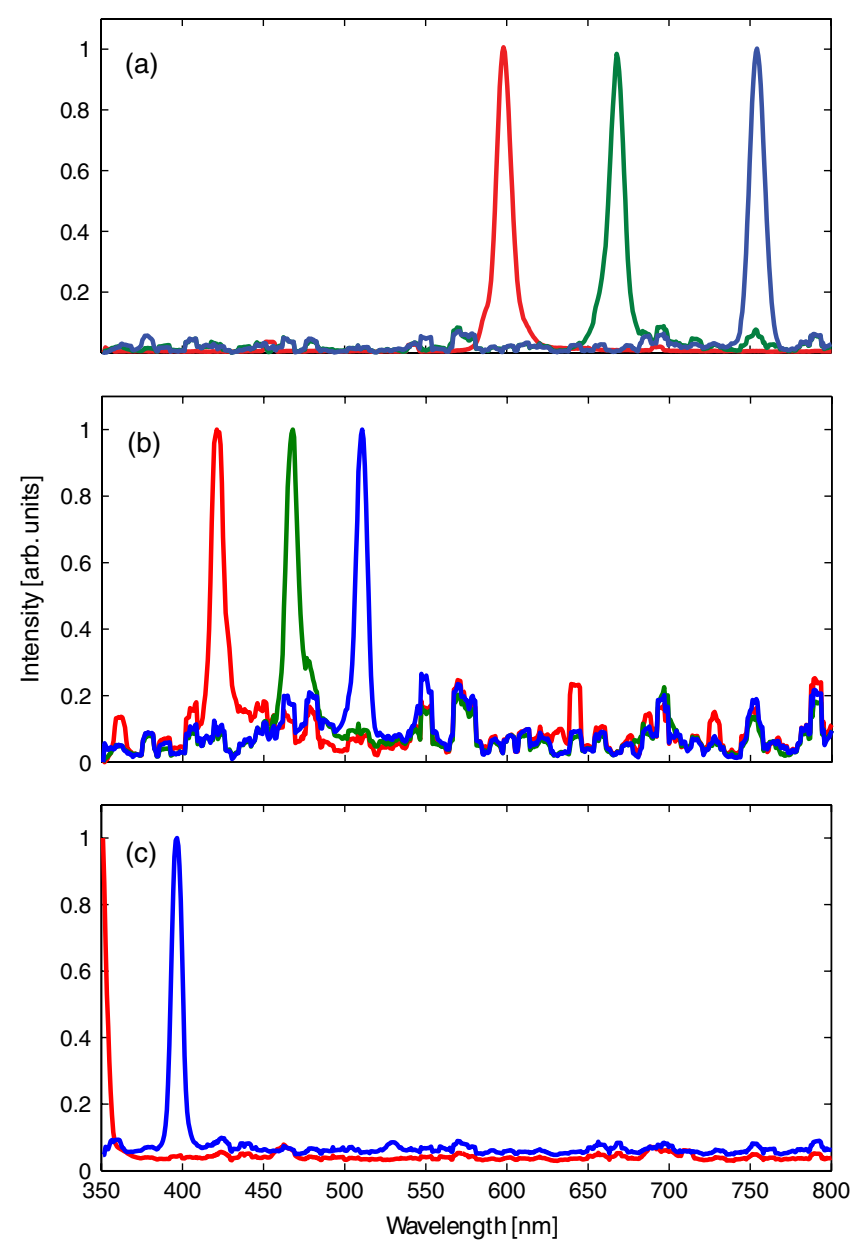

FIG. 2. Typical spectrums of FEL radiation during tuning: (a) second harmonic of the seed laser, (b) third harmonic of the seed laser, and (c) fourth harmonic of the seed laser.

flexibility can be provided by varying the beam energy at the cost of complexity of the operation.

\section{COHERENCE PROPERTIES OF THE RADIATION}

With the rapid developments of free-electron lasers in the extreme-ultraviolet (EUV) and x-ray region, the measurements of the spatial and temporal coherence properties on FEL pulses have attracted extensive attention, especially for characterizing SASE-FEL pulses [7-13]. Compared to SASE, the HGHG approach has the promising quality to produce fully-coherent light [39]. However, for a widely-tunable HGHG FEL, coherence properties of the radiation need to be confirmed while tuning within such a large wavelength range.

A Michelson interferometer is used to measure the coherence lengths at harmonics of different seed laser wavelengths. The delay time is changed by translating a movable high-reflecting mirror relative to the other fixed mirror. At each delay time, the interference patterns are recorded by a lensless camera. According to the theory of partial coherence $[40,41]$, the intensity $I(P)$ on the detector is related to the time delay $\tau$ of the two waves arriving at position $P$ and the complex degree of coherence $\gamma_{12}(\tau)$. The absolute value $\left|\gamma_{12}(\tau)\right|$ can be determined via the visibility $V$ of the interference pattern, which is defined as the following expression:

$$
V=\frac{I_{\max }-I_{\min }}{I_{\max }+I_{\min }}=2 \frac{\sqrt{I_{1} I_{2}}}{I_{1}+I_{2}}\left|\gamma_{12}(\tau)\right|
$$

where $I_{1}$ and $I_{2}$ are the intensities of the interfering partial beams and $I_{\max }$ and $I_{\min }$ are the maximum and minimum intensities of the interference fringes, respectively. In the present experiment, the interfering partial beams have the same intensity distributions in the overlapped area, so the degree of coherence $\left|\gamma_{12}(\tau)\right|$ can be obtained directly from the measured visibility $V$.

The measured visibility as a function of delay time is shown in Figs. 3(a) and 3(b) for the second and third harmonics of the seed laser with central wavelength of $1200 \mathrm{~nm}$. The visibility and the corresponding error bar at each data point are determined from 60 single-shot interference patterns. From the half width at half maximum
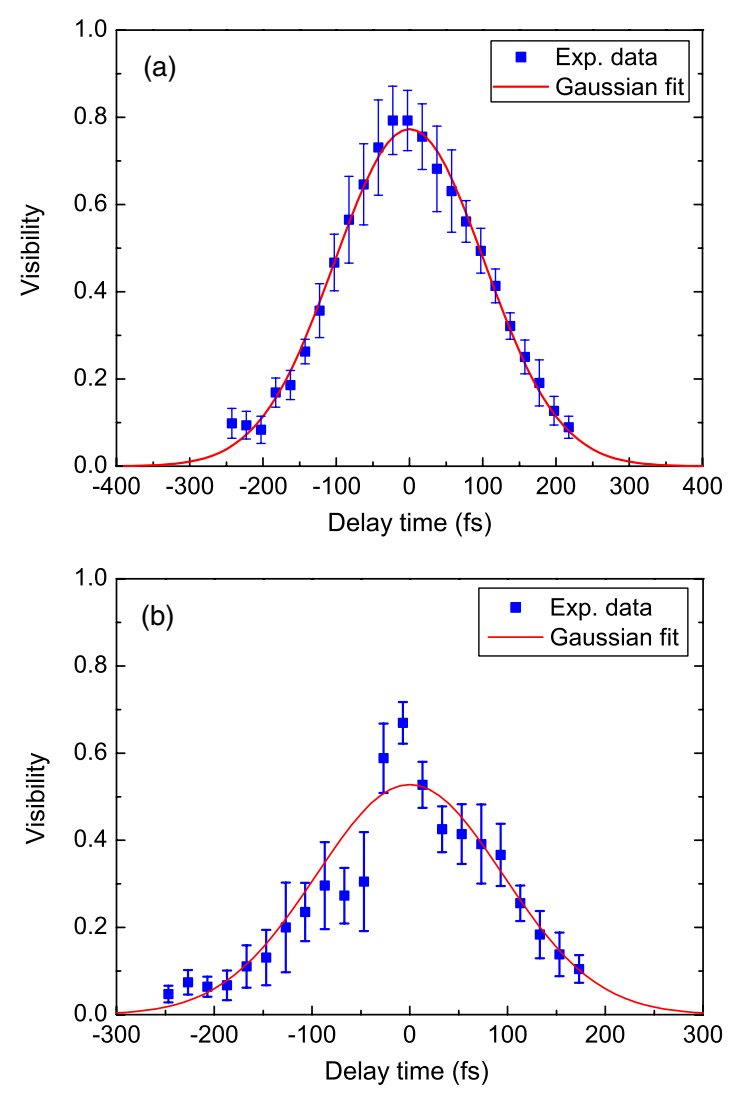

FIG. 3. Temporal coherence measurement for (a) the second harmonic and (b) the third harmonic of the $1200 \mathrm{~nm}$ seed laser. The solid curves are the Gaussian fits, which give the coherence time (HWHM) of (a) $120 \pm 2$ fs and (b) $113 \pm 6$ fs. 
(HWHM) of Gaussian fits, the coherence times are determined to be 120 and 113 fs for the second and the third harmonics, respectively. In further autocorrelation experiments on the output of the OPA, the pulse duration of the seed laser at $1200 \mathrm{~nm}$ is measured to be $82 \mathrm{fs}$. Since the Fourier transform limit for the seed laser is approximately fulfilled, the coherence time of the seed laser is assumed to be the same as the pulse duration.

The coherence length of the output light is determined by the seed laser, slippage length in the radiator, and frequency up-conversion factor [42]. According to the resonant condition, after traversing one period of the undulator, the radiation slips forward over the electron beam by the length of one radiation wavelength. For our case, the radiation wavelength is around $400-800 \mathrm{~nm}$, the total slippage in the radiator is about 100-200 fs, which is comparable with the length of the seed laser ( $82 \mathrm{fs}$, FWHM). The coherence length extension is mainly caused by the slippage effect in our experiment. Figure 4 shows the start-to-end simulation results of the pulse lengths, which compare the seed laser with radiation pulses at the entrance and exit of the radiator for different harmonic numbers. The pulse length of the 2 nd harmonic radiation at the entrance of the radiator is about $69 \mathrm{fs}$, which is shorter than the pulse length of the seed laser. This can be explained by the frequency up-conversion process. After the
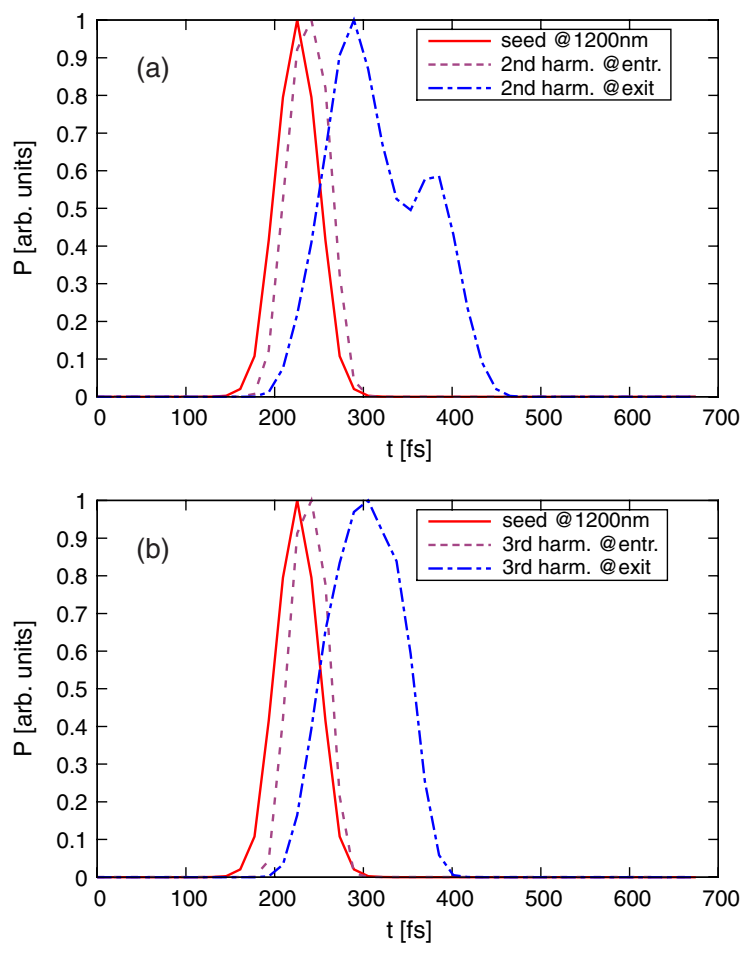

FIG. 4. Simulation of pulse lengths: (a) comparison of pulse lengths of the seed laser, 2nd harmonic radiation at the entrance and exit of the radiator; (b) comparison of pulse lengths of the seed laser, 3rd harmonic radiation at the entrance and exit of the radiator.
TABLE II. The coherence time for HGHG laser at different wavelengths of seed laser and different harmonics.

\begin{tabular}{lccc}
\hline \hline Seed wavelength $(\mathrm{nm})$ & 1200 & 1400 & 1580 \\
\hline & \multicolumn{3}{c}{ Coherence time (fs) } \\
\hline 2nd harmonic & $120 \pm 2$ & $105 \pm 4$ & $99 \pm 2$ \\
3rd harmonic & $113 \pm 6$ & $87 \pm 8$ & $92 \pm 6$ \\
4th harmonic & & & $69 \pm 5$ \\
\hline \hline
\end{tabular}

radiator, due to the slippage effect, the pulse length is extended to about 117 fs [Fig. 4(a)]. For the 3rd harmonic case, as shown in Fig. 4(b), the radiation pulse length at the entrance of the radiator is about $60 \mathrm{fs}$, which is a little shorter than the 2nd harmonic case, and it is consistent with theoretical expectation. The pulse length at the exit of the radiator is about $107 \mathrm{fs}$. It can be seen that the experimental results agree well with the simulation results.

The coherence time for the HGHG FEL are further determined for the seed laser at the wavelengths of 1400 and $1580 \mathrm{~nm}$, which are listed in Table II. Measurement results show that coherence length of the FEL output is about $69-120$ fs. Higher harmonics correspond to shorter coherence length, which agrees well with theoretical prediction. In general, the broadly tuned HGHG FEL radiation has preserved the temporal coherence of the seed.

\section{CASCADED HGHG}

To further shorten the output wavelength, the cascaded HGHG FEL together with the fresh-bunch technique [43] is considered as a promising candidate for coherent soft $\mathrm{x}$-ray sources [44]. Here we show the first results from a two-stage cascaded HGHG experiment, which is conducted at the SDUV-FEL with a new stage added [45], as shown in Fig. 5. The first stage has the same layout as being used in HGHG tuning experiments, and the main parameters of the second stage are listed in Table III. The FEL output is at the fourth harmonic of the seed laser, second harmonic at each stage.

The seed laser wavelength is chosen to be $1200 \mathrm{~nm}$ and the radiation wavelength of the first stage is $600 \mathrm{~nm}$. After the first stage, the electron beam is guided into a fresh-bunch chicane to make the first-stage radiation pulse (marked as FEL1) overlap a portion of fresh beam (marked as EB2), then FEL1 will introduce energy

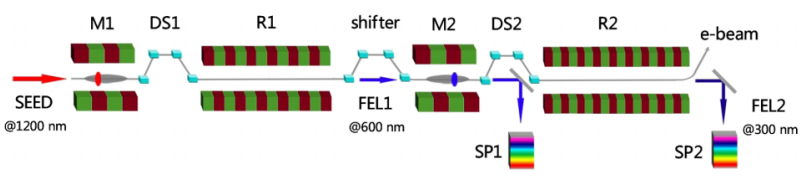

FIG. 5. Layout of the cascaded HGHG FEL experiments at the SDUV-FEL. M: modulator; DS: dispersive section; R: radiator. 
TABLE III. Main parameters of the second stage.

\begin{tabular}{lcc}
\hline \hline Parameter & Value & Unit \\
\hline Shifter for fresh bunch & & \\
Length & 2.0 & $\mathrm{~m}$ \\
Dispersive strength $\left(\mathrm{R}_{56}\right)$ & $0-2.0$ & $\mathrm{~mm}$ \\
\hline Modulator 2 & & \\
Period length & 40 & $\mathrm{~mm}$ \\
Period number & 16 & \\
Undulator parameter $K$ & 1.74 & $\mathrm{~mm}$ \\
\hline Dispersive section 2 & & $\mathrm{~mm}$ \\
Dispersive strength $\left(\mathrm{R}_{56}\right)$ & $0-4.0$ & \\
\hline Radiator 2 & & \\
Period length & 25 & \\
Period number & $60 * 6$ & \\
Undulator parameter $K$ & 1.424 & \\
\hline \hline
\end{tabular}

modulation to EB2 in the modulator of the second stage (M2). To achieve energy modulation, M2 must be tuned resonant at $600 \mathrm{~nm}$. Thus, the used portion of beam (which radiated in the first stage, marked as EB1) will continue to radiate in M2 (marking this radiation pulse as FEL1p). FEL1 and FEL1p will be separated by several hundreds of femtoseconds, depending upon the strength of the fresh-bunch chicane. The observed spectrum after M2 will have some kind of interference pattern, as shown in Fig. 6(a).

On the basis of the parameters shown in Tables I and III, start-to-end tracking of the electron beam, including all components of the SDUV-FEL, has been carried out for the cascaded HGHG experiment. The electron beam dynamics in the photoinjector was simulated with ASTRA [46] to take into account space-charge effects. ELEGANT [47] was used for the simulation in the remainder of the linac, while tracking in the undulators was performed with GENESIS [48].

The start-to-end simulation results for the bunch refreshing process are shown in Figs. 6(b) and 6(c), which are consistent with the measured spectrum [Fig. 6(a)]. The spacing between the two pulses can be easily inferred from the interference pattern, which agrees very well with the fresh-bunch chicane setting.

In the second stage, FEL1 serves as the seed and interacts with the fresh part EB2. The radiation undulators are tuned to be resonant at $300 \mathrm{~nm}$, and EB2 modulated by FEL1 will lase at $300 \mathrm{~nm}$, which is the second harmonic of FEL1. According to the simulation results, the residual bunching of EB1 is nearly smeared out by the dispersive sections, as shown in Fig. 7.

The spectrum of the second stage output and the simulation results are shown in Fig. 8. It can be seen that the start-to-end simulation results agree well with the experimental results.
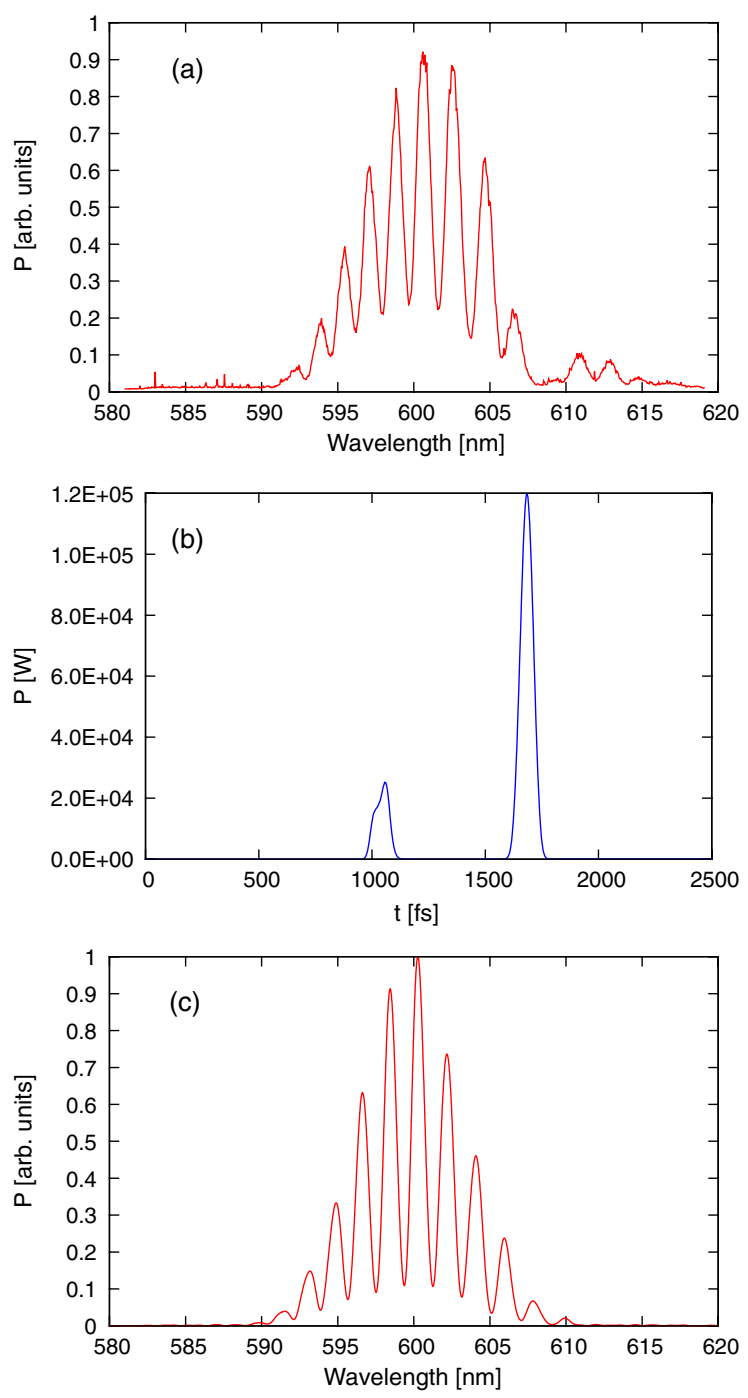

FIG. 6. Radiation pulses and corresponding spectrum after the modulator of the second stage. Two pulses have been generated by $\mathrm{R} 1$ and $\mathrm{M} 2$, which results in an interference pattern of the spectrum: (a) measured spectrum, (b) simulation result of the pulse structure, and (c) simulation result of the spectrum.

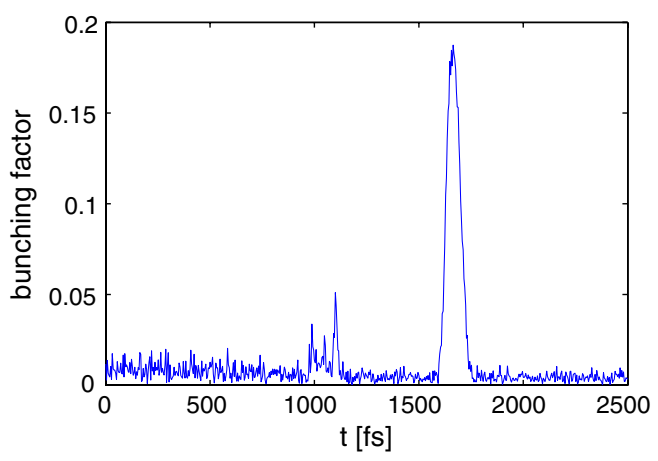

FIG. 7. Bunching factor distribution along the electron beam at the entrance to the radiator of the second stage. The microbunching in the disturbed part of the electron beam (used in the first stage) is nearly smeared out by the shifter and DS2. The bunching factor of this part is much smaller than that of the fresh part. 

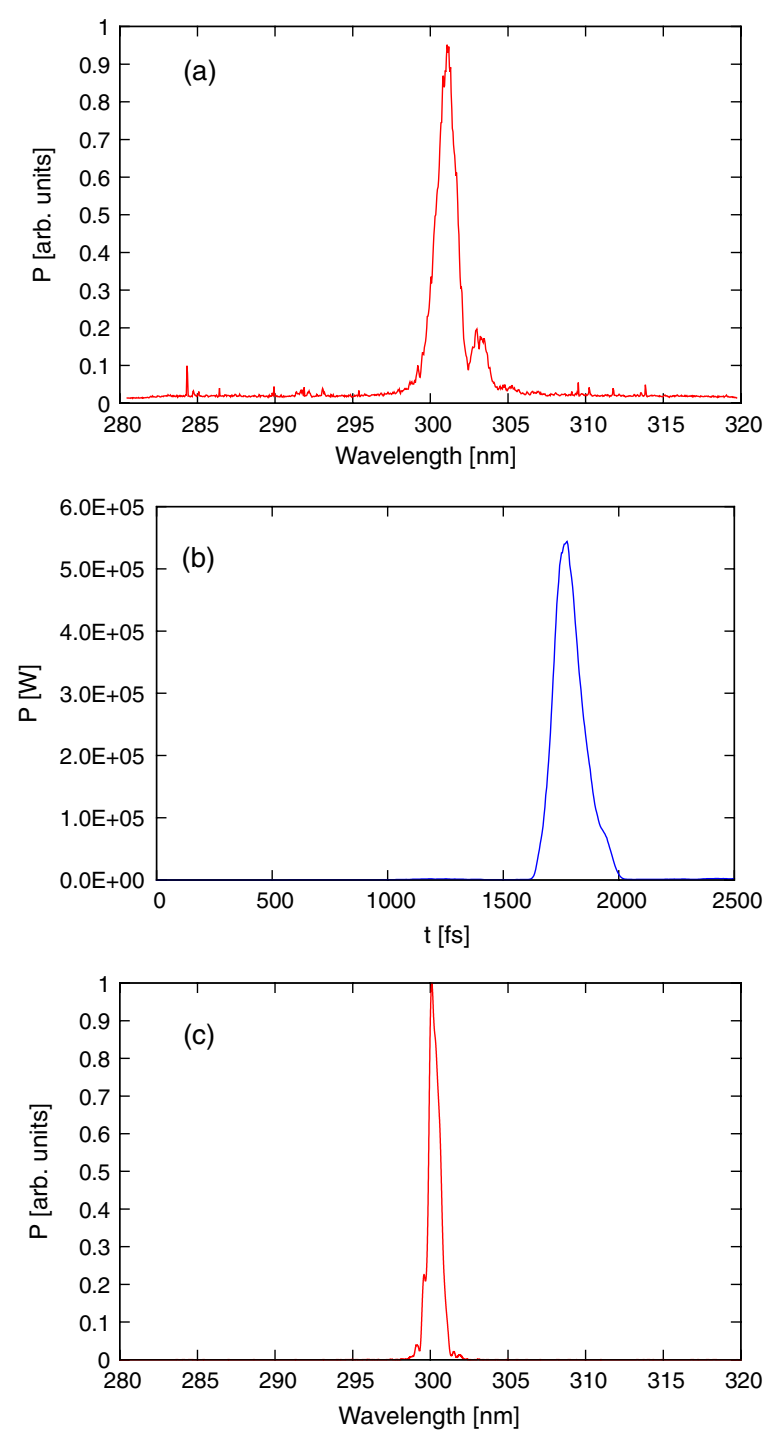

FIG. 8. Output radiation pulse and corresponding spectrum of the second stage: (a) measured spectrum, (b) simulation result of the pulse structure, and (c) simulation result of the spectrum.

\section{CONCLUSIONS}

In summary, we have demonstrated experimentally that a laser-seeded free-electron laser can provide continuous scanning of wavelengths by combining some mature laser and accelerator technologies. With OPA, variable-gap undulators and the selection of harmonics, the HGHG-type FELs can provide a wide range of wavelength tunability and fully-coherent radiation. The experimental results agree well with theoretical predictions and start-to-end simulations. We have also implemented a cascaded HGHG configuration at the SDUV-FEL. The interferencelike spectrum pattern proves that the fresh-bunch technique is valid. The coherent signal from the cascaded HGHG at the fourth harmonic of the seed is observed.

OPA technology plus sum frequency generation and second harmonic generation will make the seed laser wavelength tunable in the UV range, and then the results presented above can be easily extended to the EUV regime. Actually, based on HGHG principle, the Dalian Coherent Light Source, a fully-coherent fully-tunable EUV-FEL user facility operating in the wavelength range from 50 to $150 \mathrm{~nm}$ is already under construction [49].

It is also possible to utilize these techniques to achieve fully-coherent FELs with wavelengths in the x-ray regime. While an UV seed combined with HGHG scheme can obtain soft x-ray output in a cascaded configuration, an UV seed plus EEHG scheme should be able to achieve a soft x-ray FEL in one stage. An even more sophisticated echo-enabled staged harmonic generation [50] scheme will probably push the radiation wavelength down to hard $\mathrm{x}$-ray regime.

\section{ACKNOWLEDGMENTS}

The authors would like to thank the operation staff in the Shanghai Institute of Applied Physics for the excellent support during the SDUV-FEL experiments. We would like to thank L. H. Yu (BNL), Z. Huang (SLAC), X.F. Zhao (SINAP), S. Y. Chen (IHEP), D. H. He (NSRL), and J.E. Chen (PKU) for helpful discussions and comments. We also acknowledge support from the National 973 Program of China (Grant No. 2011CB808300) and the National Natural Science Foundation of China (Grants No. 10935011 and No. 11075118).

[1] J. Andruszkow, B. Aune, V. Ayvazyan et al., Phys. Rev. Lett. 85, 3825 (2000).

[2] W. Ackermann, G. Asova, V. Ayvazyan et al., Nat. Photonics 1, 336 (2007).

[3] P. Emma, R. Akre, J. Arthur et al., Nat. Photonics 4, 641 (2010).

[4] D. Pile, Nat. Photonics 5, 456 (2011).

[5] A. M. Kondratenko and E. L. Saldin, Part. Accel. 10, 207 (1980).

[6] R. Bonifacio, C. Pellegrini, and L. Narducci, Opt. Commun. 50, 373 (1984).

[7] R. Ischebeck, J. Feldhaus, C. Gerth et al., Nucl. Instrum. Methods Phys. Res., Sect. A 507, 175 (2003).

[8] A. Singer, I. A. Vartanyants, M. Kuhlmann, S. Duesterer, R. Treusch, and J. Feldhaus, Phys. Rev. Lett. 101, 254801 (2008).

[9] S. Roling, B. Siemer, M. Wöstmann, H. Zacharias, R. Mitzner, A. Singer, K. Tiedtke, and I. A. Vartanyants, Phys. Rev. ST Accel. Beams 14, 080701 (2011).

[10] I. A. Vartanyants, A. Singer, A. P. Mancuso et al., Phys. Rev. Lett. 107, 144801 (2011).

[11] C. Gutt, P. Wochner, B. Fischer et al., Phys. Rev. Lett. 108, 024801 (2012).

[12] R. Mitzner, B. Siemer, M. Neeb et al., Opt. Express 16, 19909 (2008).

[13] W. F. Schlotter, F. Sorgenfrei, T. Beeck, M. Beye, S. Gieschen, H. Meyer, M. Nagasono, A. Föhlisch, and W. Wurth, Opt. Lett. 35, 372 (2010). 
[14] K.-J. Kim, Nucl. Instrum. Methods Phys. Res., Sect. A 358, 31 (1995).

[15] L. H. Yu, Phys. Rev. A 44, 5178 (1991).

[16] L. H. Yu, M. Babzien, I. Ben-Zvi et al., Science 289, 932 (2000).

[17] L. H. Yu, L. DiMauro, A. Doyuran et al., Phys. Rev. Lett. 91, 074801 (2003).

[18] E. Allaria, R. Appio, L. Badano et al., Nat. Photonics 6, 699 (2012).

[19] G. Stupakov, Phys. Rev. Lett. 102, 074801 (2009).

[20] J. H. Chen, H.X. Deng, Q. Gu, D. G. Li, D. Wang, M. Zhang, and Z. T. Zhao, Chinese Phys. C 33, 706 (2009).

[21] D. Xiang and G. Stupakov, Phys. Rev. ST Accel. Beams 12, 030702 (2009).

[22] D. Xiang, E. Colby, M. Dunning et al., Phys. Rev. Lett. 105, 114801 (2010).

[23] Z. T. Zhao, D. Wang, J.H. Chen et al., Nat. Photonics 6, 360 (2012).

[24] G. Lambert, T. Hara, D. Garzella et al., Nat. Phys. 4, 296 (2008).

[25] M. Labat, M. Bellaveglia, M. Bougeard et al., Phys. Rev. Lett. 107, 224801 (2011).

[26] T. Togashi, E. J. Takahashi, K. Midorikawa et al., Opt. Express 19, 317 (2011).

[27] J. Feldhaus, E. Saldin, J. Schneider, E. Schneidmiller, and M. Yurkov, Opt. Commun. 140, 341 (1997).

[28] G. Geloni, V. Kocharyan, and E. Saldin, J. Mod. Opt. 58, 1391 (2011).

[29] J. Amann, W. Berg, V. Blank et al., Nat. Photonics 6, 693 (2012).

[30] T. Shaftan and L. H. Yu, Phys. Rev. E 71, 046501 (2005).

[31] H.X. Deng, X. T. Wang, and Z. M. Dai, Phys. Rev. ST Accel. Beams 11, 040703 (2008).

[32] H. X. Deng and Z. M. Dai, J. Phys. D 41, 115503 (2008).

[33] E. Allaria, M. Danailov, and G. D. Ninno, Europhys. Lett. 89, 64005 (2010).
[34] X. Yang, B. Podobedov, Y. Shen et al., in Conference Proceedings of FEL 2010, Malmö, Sweden.

[35] E. Allaria, G. D. Ninno, and C. Spezzani, Opt. Express 19, 10619 (2011).

[36] R. B. R. Baumgartner, IEEE J. Quantum Electron. 15, 432 (1979).

[37] R. Butkus, R. Danielius, A. Dubietis, A. Piskarskas, and A. Stabinis, Appl. Phys. B 79, 693 (2004).

[38] Z. T. Zhao, Z. M. Dai, X. F. Zhao, D. K. Liu, Q. G. Zhou, D. H. He, Q. K. Jia, S. Y. Chen, and J.P. Dai, Nucl. Instrum. Methods Phys. Res., Sect. A 528, 591 (2004).

[39] A. Doyuran, M. Babzien, T. Shaftan et al., Phys. Rev. Lett. 86, 5902 (2001).

[40] L. Mandel and E. Wolf, Optical Coherence and Quantum Optics (Cambridge University Press, Cambridge, 1995).

[41] J.W. Goodman, Statistical Optics (Wiley-Interscience, New York, 1985).

[42] D. Ratner, A. Fry, G. Stupakov, and W. White, Phys. Rev. ST Accel. Beams 15, 030702 (2012).

[43] I. Ben-Zvi, K. M. Yang, and L.H. Yu, Nucl. Instrum. Methods Phys. Res., Sect. A 318, 726 (1992).

[44] L. H. Yu and I. Ben-Zvi, Nucl. Instrum. Methods Phys. Res., Sect. A 393, 96 (1997).

[45] C. Feng, M. Zhang, G. Q. Lin, Q. Gu, H.X. Deng, J. H. Chen, D. Wang, and Z. T. Zhao, Chin. Sci. Bull. 57, 3423 (2012).

[46] K. Floettmann, ASTRA User's Manual (1999), available at http://www.desy.de/mpyflo/Astra_dokumentation.

[47] M. Borland, Technical Report No. LS-287, Argonne National Laboratory, 2000.

[48] S. Reiche, Nucl. Instrum. Methods Phys. Res., Sect. A 429, 243 (1999).

[49] D. Wang, T. Zhang, Z. T. Zhao et al., in Conference Proceedings of FEL 2011, Shanghai, China.

[50] C. Feng and Z. T. Zhao, Chin. Sci. Bull. 55, 221 (2010). 